\title{
DYNAMIC RESPONSE OF THREE-LAYERED ANNULAR PLATE WITH IMPERFECTIONS
}

\author{
DOROTA PAWLUS \\ Faculty of Mechanical Engineering and Computer Science, University of Bielsko-Biała, \\ ul. Willowa 2, 43-309 Bielsko-Biała, Poland, e-mail: doro@ath.bielsko.pl
}

\begin{abstract}
This paper presents the imperfection sensitivity of annular plate with three-layered structure. The plate composed of thin elastic facings and a thicker elastic core is loaded in facing plane. The classical issue of a three-layered plate was solved for dynamic deflection problem using the approximation methods: orthogonalization and finite difference. The solution includes the axisymmetric and asymmetric plate modes of the dynamic stability loss. The evaluation of the rate of plate sensitivity to imperfection of plate preliminary geometry has been enriched by the analysis of plate models built of finite elements. The ABAQUS program has been used. The numerous calculation results in the form of deflection characteristics, buckling modes, values of critical parameters create the view of response of dynamic plate structure with different rate of imperfection and linear in time loading growth, too.
\end{abstract}

Key words: sandwich, annular plate, dynamic stability, imperfections, finite difference method, finite element method

\section{INTRODUCTION}

In this paper, the problem of structure imperfection sensitivity will be presented for the element of a three-layered annular plate under time-varying load acting in facings plane. Two ways of solving the problem of plate dynamic deflection using the approximation methods: finite difference and finite element will be proposed. The solutions include both forms of plate loss of dynamic stability: elementary axisymmetric and asymmetric circumferentially waved. Adjusting asymmetric buckling modes in solution of examined plate the analytical and numerical solution becomes more complicated. However, it is essential in aiming to evaluate the critical parameters properly. The evaluation of critical state of the three-layered plate analysed with elastic facings and a core has been presented in [8], [9]. A detailed description of complex analytical and numerical solution of dynamic deflection problem has been presented in [7].

This work is focused on showing the imperfection sensitivity of plate to its dynamic response.

It should be noticed that the problem of dynamic stability of sandwich structure of annular plate with elastic or viscoelastic core with damping properties is still being undertaken [1]-[3], [14], [15]. This problem is the subject of constant numerical analyses but this work, in the author's opinion, adds some new elements to the knowledge of the field under investigation.

A full analysis of dynamic critical state of construction element requires the evaluation of the influence of many parameters describing the material, geometry, loading and initially-edge conditions on response of an object examined. It is a complex multiparameter problem. The analysis of the influence of preliminary geometry state of structure has particular meaning. In reality, its spatial shape has irregular character in many cases difficult to describe. The meaning of imperfection analysis in investigation of buckling structures increases in dynamic problems. The small variations of the rate of preliminary structure deformation essentially change the values of critical dynamic loads. For real objects these values could be significantly lower than those calculated in model examinations. Hence, the evaluation of the influence of preliminary deflection of examined element - here, sandwich annular plate - on its behaviour in the field of dynamic buckling phenomenon has the basic meaning.

\section{PROBLEM FORMULATION}

A three-layered annular plate with elastic core loaded in the plane of facings is taken into considera- 
tion. The classical three-layered transverse structure is composed of elastic thin facings and suitably thicker soft core (no. 1, 3 and 2 in Fig. 1, respectively). A scheme of the plate analysed is presented in Fig. 1.

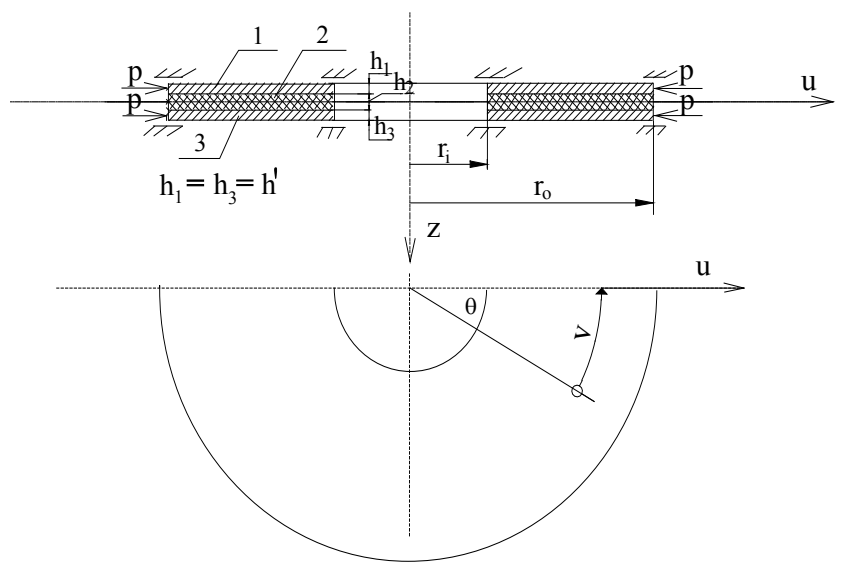

Fig. 1. Scheme of the plate analysed

The outer plate layers are loaded on inner or outer edge with uniformly distributed compressed stress proportionally increasing with time $t$ and velocity $s$. The formula is the following

$$
p=s t
$$

where $p$ - compressed stress, $s$ - rate of plate loading, $t$ - time.

The dynamic critical parameters of the plate are observed for both axially-symmetrical form of plate buckling and circumferentially waved ones. The importance of waved buckling forms occurs for the plate case loaded on outer edge. The values of critical dynamic loads are significantly lower than the value calculated for the axially-symmetrical form.

The parameters presented in Fig. 1 and the form and rate of plate preliminary deflection are important in the description of the plate geometry. The plate supported in slidably clamped edges is analysed.

Evaluating the influence of the plate imperfections on the values of dynamic critical loads and additional deflections the ideal flat surface of plate has been distorted. The flat surface is replaced by the axiallysymmetrical surface with the various rate of preliminary deflection in the case of plate loaded on inner edge and by the shape composed of circumferentially waved form and axially-symmetrical one in the case of plate compressed on the outer edge. The number of waves in circumferential plate direction is denoted by $m$. An axially-symmetrical form as the component of the total plate preliminary deflection could be bent to different degree. The mathematical description of this complex form of preliminary deflection $\zeta_{0}$ is expressed by equation (3).

Figure 2 shows the change of the shape of plate's preliminary deflection from the form with $m=5$ circumferentially waved (Fig. 2a) to the form distorted by the additional axially-symmetrical form (Fig. 2b) obtained using equation (3) for the numbers: $\xi_{1}=5$, $\xi_{2}=1, m=5$.

The criterion presented in [13] has been adopted as the condition of the loss of plate stability. According to this criterion the loss of plate stability occurs at the moment of time when the speed of the point of maximum deflection reaches the first maximum value.

Figure 3 explains the means of determination of the value of critical time and deflection.

(a)

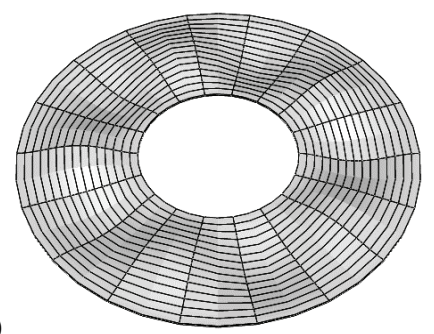

(b)

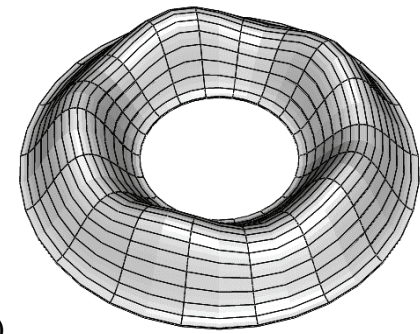

Fig. 2. The forms of preliminary plate deflections: (a) expressed by the number $m=5$ waves, (b) complex form built of axially-symmetrical form for $\xi_{1}=5$ and waved form expressed by $m=5$ and $\xi_{2}=1$ obtained for the numbers $\xi_{1}, \xi_{2} 200$ times magnified for better visualization

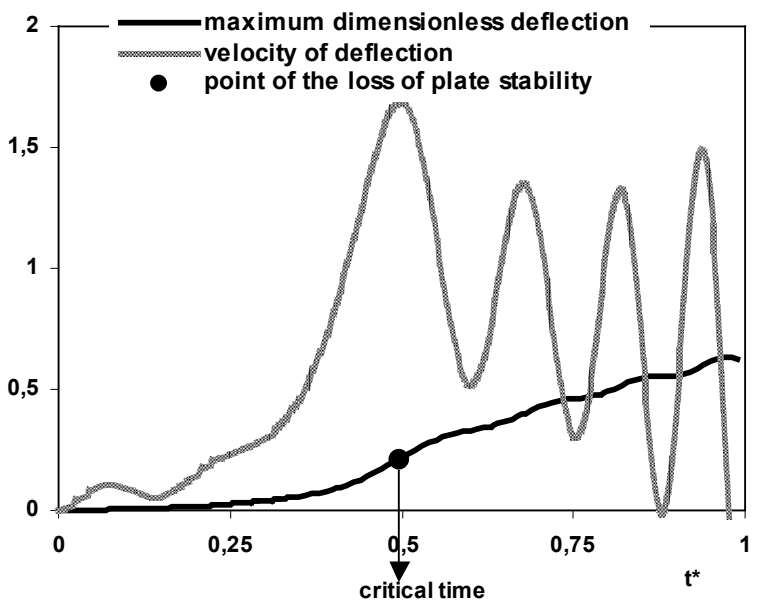

Fig. 3. The example lines of time histories of deflection and velocity of deflection of three-layered plate loaded on inner edge 
The plate dynamic critical state is characterized by values of critical load $p_{c r d y n}$, critical time $t_{c r}$ and deflection $y_{c r}$. In the evaluation of calculated critical dynamic and static loads the minimal values are important. It is worth noticing that in the case of the analysed plate loaded on the inner edge the loss of the static and dynamic stability is observed in the axially-symmetrical form for the minimal critical loads [7]. Figure 4 shows the form of plate loading and buckling form.

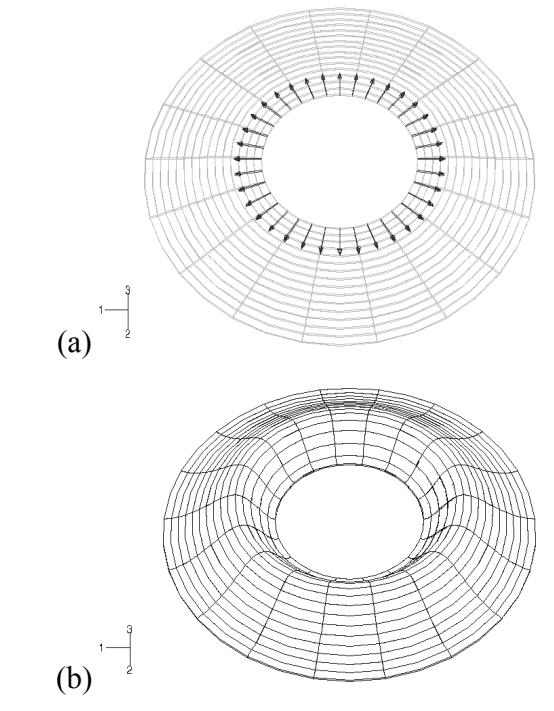

Fig. 4. Plate loading (a) and axially-symmetrical buckling form (b)

\section{PROBLEM SOLUTION}

The problem has been solved using two approximation methods: finite difference method (FDM) and finite element method (FEM). The assumptions of the classical theory [12] of sandwich plates with the broken line hypothesis and the distribution of the plate layers in carrying the stresses: normal by the facings and the shear one by the core have been accepted in building the plate computational models by:

- the suitable expressions using the FDM solution,

- selection of the mesh elements in building the FEM plate model.

\subsection{BASIC EQUATIONS IN FDM SOLUTION}

A detailed solution with all the equation forms and the description of algebraic operations is presented in
[7]-[9]. The main elements of the solution are the following:

- formulation of the dynamic equilibrium equations for each plate layer,

- description of the core deformation in radial and circumferential directions,

- application of the linear physical relations of Hook's law,

- establishing the sectional forces and moments in facings using the equations of nonlinear Kármán's plate,

- formulation of the resultant, transverse radial and circumferential forces and the resultant membrane forces expressed by the stress function introduced,

- determination of the initial, loading and boundary conditions:

$$
\begin{gathered}
w_{\left.d\right|_{t=0}}=0,\left.\quad w_{d, t}\right|_{t=0}=0, \\
\left.\sigma_{r}\right|_{r=r_{i}}=-p(t) d_{1},\left.\quad \sigma_{r}\right|_{r=r_{o}}=-p(t) d_{2}, \\
\left.\tau_{r \theta}\right|_{r=r_{i}\left(r_{o}\right)}=0, \\
\left.w\right|_{r=r_{i}\left(r_{o}\right)}=0,\left.\quad w_{r}\right|_{r=r_{i}\left(r_{o}\right)}=0, \\
\delta=\left.\gamma\right|_{r=r_{i}\left(r_{o}\right)}=0,\left.\quad \delta_{r_{r}}\right|_{r=r_{i}\left(r_{o}\right)}=0,
\end{gathered}
$$

where

$w$ - total deflection, $w_{d}$ - additional deflection,

$\sigma_{r}$ - radial stress, $\tau_{r \theta}-$ shear stress,

$d_{1}, d_{2}$ - quantities, equal to 0 or 1 , determining the loading of the inner or/and outer plate perimeter,

$\delta, \gamma$-differences of radial and circumferential displacements of the points in middle surfaces of facings $\delta=u_{3}-u_{1}, \gamma=v_{3}-v_{1}$, respectively,

- assumption of the form of predeflection $w_{o}$ according to the formula [11], [16]

$$
\zeta_{o}(\rho, \theta)=\xi_{1}(\rho) \eta(\rho)+\xi_{2}(\rho) \eta(\rho) \cos (m \theta)
$$

where

$\zeta_{o}, \rho-$ dimensionless quantities of predeflection $\zeta_{o}=\frac{w_{o}}{h}$ and plate radius $\rho=\frac{r}{r_{o}}$, respectively ( $h$ - total plate thickness),

$\xi_{1}, \xi_{2}-$ calibrating numbers,

$\eta(\rho)$ - function expressed by the equation

$$
\eta(\rho)=\rho^{4}+A_{1} \rho^{2}+A_{2} \rho^{2} \ln \rho+A_{3} \ln \rho+A_{4},
$$

$A_{i}$ - quantities fulfilling the conditions of clamped plate edges, $i=1,2,3,4$, 
$m$ - number of buckling waves in circumferential plate direction,

- determination of the basic differential equation expressing the deflections of the analysed sandwich plate

$$
\begin{gathered}
N_{1} w_{d, r r r}+\frac{2 N_{1}}{r} w_{d, r r r}-\frac{N_{1}}{r^{2}} w_{d, r r}+\frac{N_{1}}{r^{3}} w_{d, r}+\frac{N_{1}}{r^{4}} w_{d, \theta \theta \theta \theta} \\
+\frac{2\left(N_{1}+N_{2}\right)}{r^{4}} w_{d, \theta \theta}+\frac{2 N_{2}}{r^{2} w_{d, r r \theta \theta}}-\frac{2 N_{2}}{r^{3}} w_{d, r \theta \theta} \\
-G_{2} \frac{H^{\prime}}{h_{2}} \frac{1}{r}\left(\gamma_{, \theta}+\delta+r \delta_{, r}+H^{\prime} \frac{1}{r} w_{d, \theta \theta}+H^{\prime} w_{d, r}+H^{\prime} r w_{d, r r}\right) \\
=\frac{2 h^{\prime}}{r}\left(\frac{2}{r^{2}} \Phi_{, \theta} w_{, r \theta}-\frac{2}{r} \Phi_{, r \theta} w_{, r \theta}+\frac{2}{r^{2}} w_{, \theta} \Phi_{, r \theta}-\frac{2}{r^{3}} \Phi_{, \theta} w_{, \theta}\right. \\
\left.+w_{, r} \Phi_{, r r}+\Phi_{, r} w_{, r r}+\frac{1}{r} \Phi_{, \theta \theta} w_{, r r}+\frac{1}{r} \Phi_{, r r} w_{, \theta \theta}\right)-M w_{d, t t} .
\end{gathered}
$$

Introducing the dimensionless quantities, expressions and shape functions for the additional plate deflection $\zeta_{1}$, stress function $F$ [11] and differences $\bar{\delta}, \bar{\gamma}$

$$
\begin{gathered}
\zeta_{1}=\frac{w_{d}}{h}, \quad F=\frac{\Phi}{E h^{2}}, \quad \bar{\delta}=\frac{\delta}{h}, \quad \bar{\gamma}=\frac{\gamma}{h}, \\
\zeta_{1}(\rho, \theta, t)=X_{1}(\rho, t) \cos (m \theta), \\
F(\rho, \theta, t)=F_{a}(\rho, t)+F_{b}(\rho, t) \cos (m \theta) \\
+F_{c}(\rho, t) \cos (2 m \theta), \\
\bar{\delta}(\rho, \theta, t)=\bar{\delta}(\rho, t) \cos (m \theta), \\
\bar{\gamma}(\rho, \theta, t)=\bar{\gamma}(\rho, t) \sin (m \theta),
\end{gathered}
$$

where

$\Phi-$ stress function, $E$ - Young's modulus of facing material,

after use of the orthogonalization method for elimination of the angular variable $\theta$ equation (4) has been replaced by

$-\mathrm{WK} 1 \cdot X_{1, \rho \rho \rho \rho}-\frac{2 \mathrm{WK} 1}{\rho} X_{1, \rho \rho \rho}+\frac{\mathrm{WK} 3}{\rho^{2}} X_{1, \rho \rho}-\frac{\mathrm{WK} 3}{\rho^{3}} X_{1, \rho}$

$$
-\frac{\mathrm{WK} 4}{\rho^{4}} X_{1}+\frac{2 \mathrm{WK}}{\rho^{4}} m^{2} X_{1}+\frac{\mathrm{WK} 5}{\rho} H^{\prime}
$$

$\left(m \bar{\gamma}+\rho \cdot \bar{\delta}_{, \rho}+\bar{\delta}-\frac{m^{2}}{\rho} \frac{H^{\prime}}{r_{o}} X_{1}+\frac{H^{\prime}}{r_{o}} X_{1, \rho}+\frac{H^{\prime}}{r_{o}} X_{1, \rho \rho}\right)$

$$
+\frac{2 \mathrm{WK} 5^{2} \mathrm{WK} 2}{\rho}\left(X_{, \rho} \cdot Y_{, o \rho}+Y_{o} \cdot X_{, \rho \rho}-\frac{m^{2}}{\rho} X \cdot Y_{o, \rho}\right)
$$

$$
\begin{gathered}
+\left(1-\delta_{m}^{o}\right)\left\{\frac{4 \mathrm{WK} 5^{2} \mathrm{WK} 2}{\rho^{2}}\right. \\
\times\left(\frac{1}{\rho} m^{2} F_{c} X_{, \rho}-F_{c, \rho} m^{2} X_{, \rho}+\frac{1}{\rho} F_{c, \rho} m^{2} X-\frac{1}{\rho^{2}} F_{c} m^{2} X\right) \\
+\frac{\mathrm{WK} 5^{2} \mathrm{WK} 2}{\rho}\left(2 X_{a, \rho} F_{b, \rho \rho}+X_{, \rho} F_{c, \rho \rho}+2 X_{a, \rho \rho} F_{b, \rho}\right. \\
\left.\left.+X_{, \rho \rho} F_{c, \rho}-\frac{2}{\rho} m^{2} F_{b} X_{a, \rho \rho}-\frac{4}{\rho} m^{2} F_{c} X_{, \rho \rho}-\frac{1}{\rho} m^{2} F_{c, \rho \rho} X\right)\right\} \\
=\mathrm{K}^{2} \cdot \mathrm{WK} 5 \cdot \mathrm{WK} 8 \cdot X_{1_{, * *} *}
\end{gathered}
$$

where

$\delta_{m}^{0}-$ Kronecker's symbol,

$X(\rho, t)=X_{1}(\rho, t)+X_{b}(\rho), X_{b}(\rho)=\xi_{2} \eta(\rho), Y_{o}=F_{a, \rho}$,

$t^{*}=t \cdot \mathrm{K} 7, \mathrm{~K} 7=\frac{s}{p_{c r}}\left(p_{c r}-\right.$ critical, static stress $)$,

WK1, WK2, WK3, WK4, WK5, WK8 - quantities expressed by the geometric and material parameters of plate layers and by the number $m$.

The quantities $\bar{\delta}$ and $\bar{\gamma}$ in equation (7) have been calculated using the additional equilibrium equations for forces acting on the undeformed outer plate layers in the $u$ and $v$ directions (Fig. 1). The components $F_{a}$, $F_{b}, F_{c}$ of the stress function have been found on the strength of the three equations obtained from the equation

$$
\begin{gathered}
\Phi_{, r r r r}+\frac{2}{r} \Phi_{, r r r}-\frac{1}{r^{2}} \Phi_{, r r}+\frac{1}{r^{3}} \Phi_{, r}+\frac{1}{r^{4}} \Phi_{, \theta \theta \theta \theta} \\
+\frac{4}{r^{4}} \Phi_{, \theta \theta}-\frac{2}{r^{3}} \Phi_{, r \theta \theta}+\frac{2}{r^{2}} \Phi_{, r r \theta \theta} \\
=E\left[\frac{1}{r} w_{o_{, r r}}\left(w_{o_{, r}}+\frac{1}{r} w_{o_{\theta \theta}}\right)-\frac{1}{r^{2}}\left(\frac{1}{r} w_{o_{\theta}}-w_{o_{r \theta}}\right)^{2}\right. \\
\left.-\frac{1}{r} w_{, r r}\left(w_{, r}+\frac{1}{r} w_{, \theta \theta}\right)+\frac{1}{r^{2}}\left(\frac{1}{r} w_{, \theta}-w_{, r \theta}\right)^{2}\right]
\end{gathered}
$$

after some algebraic operation and comparison of the expressions standing by the same trigonometric functions. Equation (8) has been obtained using the relations for radial and circumferential normal forces and shearing forces in facings and after the elimination of the quantities described by the sums of the radial $\left(u_{3}+u_{1}\right)$ and the circumferential $\left(v_{3}+v_{1}\right)$ facing displacements.

The basic system of differential equations, determined using the finite difference method approximating the derivatives with respect to $\rho$ by the central 
differences at the discrete points has the following form

$$
\begin{gathered}
\mathbf{P}_{L} \mathbf{U}+\mathbf{Q}_{L}=\mathbf{K} \cdot \ddot{\mathbf{U}}, \\
\mathbf{M}_{D} \mathbf{D}=\mathbf{M}_{U} \mathbf{U}+\mathbf{M}_{G} \mathbf{G}, \\
\mathbf{M}_{G G} G=\mathbf{M}_{G U} \mathbf{U}+\mathbf{M}_{G D} D, \\
\mathbf{M}_{Y} \mathbf{Y}=\mathbf{Q}_{Y}, \\
\mathbf{M}_{V} \mathbf{V}=\mathbf{Q}_{V}, \\
\mathbf{M}_{Z} \mathbf{Z}=\mathbf{Q}_{Z},
\end{gathered}
$$

where

$$
\mathrm{K}=\mathrm{K} 7^{2} \cdot \mathrm{WK} 5 \cdot \mathrm{WK} 8 \text {, }
$$

$\mathbf{U}, \mathbf{Y}, \mathbf{V}, \mathbf{Z}$ - vectors of the plate's additional deflections and components $F_{a}, F_{b}, F_{c}$ of the stress function $F_{a_{, \rho}}=y, F_{b}=v, F_{c}=z$, respectively,

$\mathbf{Q}_{L}, \mathbf{Q}_{Y}, \mathbf{Q}_{V}, \mathbf{Q}_{Z}-$ vectors of expressions composed of the initial and additional deflections, geometric and material parameters, components of the stress function, radius $\rho$, quantity $b$ ( $b$ - length of the interval in the finite difference method), coefficients $\delta, \gamma$ and number $m$,

$\mathbf{P}_{L}, \mathbf{M}_{D}, \mathbf{M}_{U}, \mathbf{M}_{G}, \mathbf{M}_{G G}, \mathbf{M}_{G U}, \mathbf{M}_{G D}, \mathbf{M}_{Y}, \mathbf{M}_{V}, \mathbf{M}_{Z}-$ matrices of elements composed of plate parameters, quantity $b$ and number $m$,

D, $\mathbf{G}$ - vectors of expressions composed of coefficients $\delta$ and $\gamma$, respectively.

The Runge-Kutta's integration method for the initial state of plate has been used in the solution of the system of equations presented.

Equations (9)-(14) are the solution to the formulated problem of three-layered annular plate with wavy forms of buckling.

Neglecting the inertial components, initial deflections and the components $F_{b}, F_{c}$ of stress function $F$, which is the solution to the disk state, in equation (7) and in equilibrium equations of forces in facing planes the eigenvalue problem has been formulated. The calculated minimal value is the value of critical static stress $p_{c r}$ important for the static problem analysed.

\subsection{PLATE MODELS BUILT IN FEM}

Two kinds of plate models were built using the finite element method: plate model in the shape of full annulus (in this paper, called a basic model) presented in Fig. 5 and plate model built of the axisymmetric elements (called a simplistic model) presented in Fig. 6.
Computational analyses of the three-layered, annular plates compressed on the inner perimeter show the axially-symmetrical form of plate buckling corresponding to the minimal values of critical load [7]. In such a case, the simple plate model built of axisymmetric elements could be used in numerical investigations.

In selection of mesh elements the remarks presented in work [4] were used. The facing mesh is built of shell elements but the core mesh is composed of solid elements. The outer surfaces of facing mesh elements have been connected with the outer surfaces of core elements using the surface contact interaction. All parameters describing the plate model solved in finite difference method are consistent with the values of FEM model parameters.

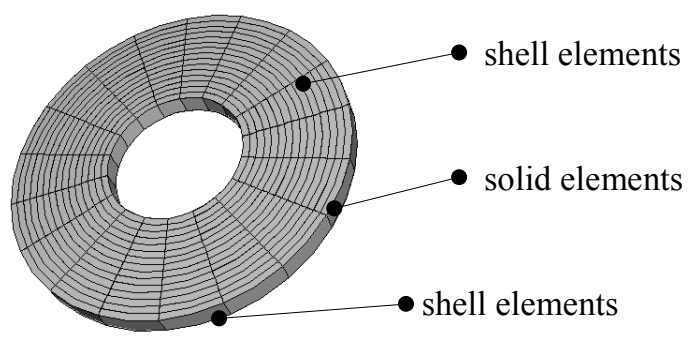

Fig. 5. Basic plate model

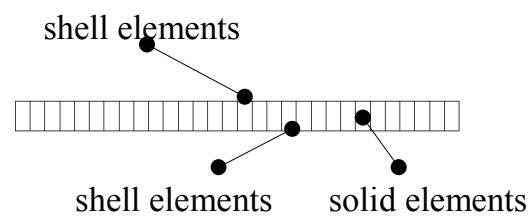

Fig. 6. Simplistic plate model

The calculations were carried out at the Academic Computer Center CYFRONET-CRACOW (KBN/SGI ORIGIN 2000/PLodzka/030/1999) using the ABAQUS system.

\section{EXEMPLARY PLATE PARAMETERS}

Exemplary numerical calculations were carried out for a plate with the following geometrical and material parameters:

- inner radius $r_{i}=0.2 \mathrm{~m}$,

- outer radius $r_{o}=0.5 \mathrm{~m}$,

- facing thickness $h^{\prime}=0.001 \mathrm{~m}$, 
- core thickness $h_{2}=0.005 \mathrm{~m}, 0.01 \mathrm{~m}, 0.02 \mathrm{~m}$, treated as the middle core thickness and $h_{2}=0.06 \mathrm{~m}$ as thick one,

- steel facing material: Youngs's modulus $E=$ $2.1 \cdot 10^{5} \mathrm{MPa}$, Poisson's ratio $v=0.3$, mass density $\mu=7.85 \cdot 10^{3} \mathrm{~kg} / \mathrm{m}$,

- polyurethane foam as core material: Kirchhoff's modulus $G_{2}=5 \mathrm{MPa}$, mass density $\mu_{2}=64 \mathrm{~kg} / \mathrm{m}^{3}$ [5] or $G_{2}=15.82 \mathrm{MPa}, \mu_{2}=93.6 \mathrm{~kg} / \mathrm{m}^{3}$ [10].

Calculated Young's moduli are the following: $E_{2}=$ $13 \mathrm{MPa}, E_{2}=41.13 \mathrm{MPa}$, respectively. They have been obtained treating the foam material as isotropic for the value of Poisson's ratio equal to $v=0.3$. Plate preliminary deflection is expressed by the values of the following parameters (see equation (3)): $\xi_{1}=0,5$, $10, \xi_{2}=0.125,0.25,0.5,1,2$ and number of waves in circumferential plate direction is consistent with the number of expected buckling waves $m$. Rapidly increasing loading acting on the plate edge is expressed by equation (1). The rate of plate loading growth $s$ is the result of the following equation: $s=\mathrm{K} 7 p_{c r}$. The value of the parameter $\mathrm{K} 7$ is equal to: $\mathrm{K} 7=20 \mathrm{1} / \mathrm{s}$. Some results showing the plate dynamic response for higher value of K7 (K7 = 40 1/s) and smaller value of the rate of loading growth $\mathrm{K} 7(\mathrm{~K} 7=101 / \mathrm{s})$ are presented, too. The adopted values of critical stress $p_{c r}$ calculated solving the eigenproblem are following: $p_{c r}$
$=217.32 \mathrm{MPa}$ obtained for plate loaded on inner edge with parameters: $G_{2}=15.82 \mathrm{MPa}, h_{2}=0.01 \mathrm{~m}, h^{\prime}=$ $0.001 \mathrm{~m}, m=0$ and $p_{c r}=46.58 \mathrm{MPa}$ obtained for plate loaded on outer perimeter with parameters: $G_{2}=15.82$ $\mathrm{MPa}, h_{2}=0.005 \mathrm{~m}, h^{\prime}=0.001 \mathrm{~m}, m=7$.

\section{DISCUSSION OF RESULTS}

The calculations using FDM method have been preceded by the analysis of the accuracy of values of dynamic critical loads $p_{c r d y n}$ for different numbers $N$ of discrete points: $N=11,14,17,21,26$. Table 1 shows the values of $p_{\text {crdyn }}$ for two plate cases. Number $N=14$, for which the FDM calculations were carried out, fulfils the accuracy up to $5 \%$ of technical error.

Table 1. Dynamic critical loads $p_{c r d y n}$ for different numbers $N$ of two plate cases: I - plate loaded on inner edge with parameters: $G_{2}=5 \mathrm{MPa}, h_{2}=0.02 \mathrm{~m}, m=0, \xi_{1}=0, \xi_{2}=2$,
$\mathrm{II}-$ plate loaded on outer edge with parameters:
$G_{2}=5 \mathrm{MPa}, h_{2}=0.005 \mathrm{~m}, m=7, \xi_{1}=5, \xi_{2}=1$

\begin{tabular}{|c|c|c|c|c|c|}
\hline \multirow{2}{*}{$\begin{array}{c}\text { Plate } \\
\text { case }\end{array}$} & \multicolumn{5}{|c|}{$N$} \\
\cline { 2 - 6 } & 11 & 14 & 17 & 21 & 26 \\
\hline I & 154.96 & 154.96 & 154.09 & 153.65 & 153.22 \\
\hline II & 24.83 & 24.08 & 23.99 & 23.80 & 23.71 \\
\hline
\end{tabular}

Table 2. The values of critical, dynamic $p_{c r d y n}$ and static $p_{c r}$ loads of plates with various rate of preliminary deflection $\xi_{2}$, loaded on inner edge

\begin{tabular}{|c|c|c|c|c|c|c|}
\hline \multirow{2}{*}{$G_{2}[\mathrm{MPa}] / h_{2}[\mathrm{~m}]$} & \multicolumn{5}{|c|}{$p_{\text {crdyn }}[\mathrm{MPa}]$} & $p_{c r}$ \\
\cline { 2 - 6 } & $\xi_{2}=0.125$ & $\xi_{2}=0.25$ & $\xi_{2}=0.5$ & $\xi_{2}=1$ & $\xi_{2}=2$ & {$[\mathrm{MPa}]$} \\
\hline $5 / 0.005$ & 112.36 & 108.01 & 103.66 & 98.88 & 92.80 & 75.61 \\
\hline $5 / 0.02$ & 174.51 & 169.73 & 164.52 & 159.30 & 154.95 & 150.29 \\
\hline $15.82 / 0.005$ & 180.16 & 176.25 & 171.90 & 166.69 & 161.47 & 149.34 \\
\hline $15.82 / 0.02$ & 359.24 & 355.33 & 350.98 & 346.64 & 343.15 & 338.94 \\
\hline
\end{tabular}

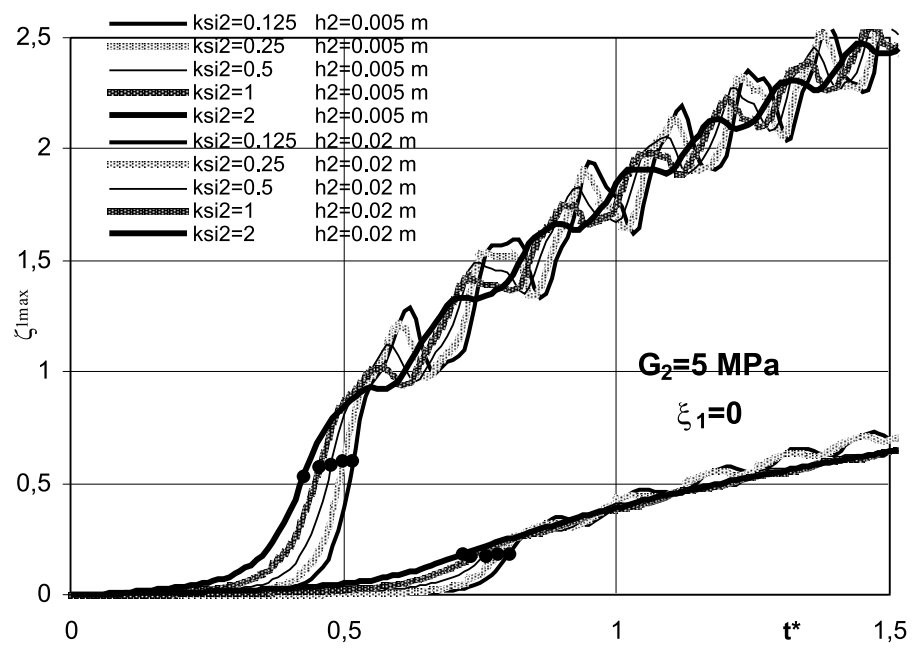

Fig. 7. The time histories of deflections of plate compressed on inner perimeter with various values of parameter $\xi_{2}$ 
The values of critical dynamic loads $p_{c r d y n}$ of plates with various rate of preliminary deflection, expressed by the value $\xi_{2}$, calculated using FDM method and compressed on the inner perimeter are presented in Table 2.

Axisymmetric mode of the loss of plate dynamic stability was analysed. The form of predeflection is in the same shape. The value of the rate of loading growth is equal to $\mathrm{K} 7=20 \mathrm{1} / \mathrm{s}$.

The exemplary characteristics presented in Fig. 7 show the differences between the deflection time histories of plates variously predeflected. Presented results show the significant decrease in values of critical dynamic loads of plates with the large rate of predeflection. The differences in values of load $p_{\text {crdyn }}$ between the plates with values $\xi_{2}$ equal to $\xi_{2}=0.125$ and $\xi_{2}=2$ are relevant and important in analysis of stability problem. The values of critical static load $p_{c r}$ are the good reference point in presented evaluation. The tendency to decay of the supercritical vibrations initiated by the increasing load for plates with the high value of parameter $\xi_{2}$ is observed, too.
The results obtained for basic and simplistic FEM model of plate confirm the sensitivity of the plate to the rate of preliminary deflection. Table 3 shows the values of critical dynamic $p_{c r d y n}$ and static $p_{c r}$ loads.

Presented in Table 3 the values of critical, static loads obtained for basic and simplistic model show good compatibility of the results calculated for the two FEM plate models analysed. This also indicates that the FEM plate models presented are built correctly.

Figure 8 shows example time histories of deflection, velocity of deflection and buckling form of FEM simplistic plate models with the parameters: $G_{2}=5 \mathrm{MPa}$, $h_{2}=0.02 \mathrm{~m}, \xi_{2}=0.125$ and $\xi_{2}=2$. The plate with high predeflection loses its dynamic stability quicker for less value of dynamic critical load without the supercritical vibrations.

The values of critical dynamic loads $p_{\text {crdyn }}$ of plates with wavy forms of buckling, compressed on outer perimeter, are shown in Fig. 9. The results presented are for plate models with preliminary deflection expressed by calibrating numbers $\xi_{1}, \xi_{2}$ (see equation (3)) equal to: $\xi_{1}=0,5,10$ and $\xi_{2}=1$.

Table 3. Values of critical dynamic $p_{c r d y n}$ and static $p_{c r}$ loads of basic and simplistic FEM plate models with different value of parameter $\xi_{2}$

\begin{tabular}{|c|c|c|c|c|c|c|c|}
\hline \multirow{4}{*}{$G_{2}[\mathrm{MPa}] / h_{2}[\mathrm{~m}]$} & \multicolumn{5}{|c|}{$p_{c r d y n}[\mathrm{MPa}]$} & \multirow{2}{*}{\multicolumn{2}{|c|}{$p_{c r}[\mathrm{MPa}]$}} \\
\hline & \multirow{2}{*}{\multicolumn{2}{|c|}{$\frac{\text { basic model }}{\xi_{2}}$}} & \multirow{2}{*}{\multicolumn{3}{|c|}{$\begin{array}{c}\text { simplistic model } \\
\xi_{2}\end{array}$}} & & \\
\hline & & & & & & \multirow{2}{*}{$\begin{array}{l}\text { basic } \\
\text { model }\end{array}$} & \multirow{2}{*}{$\begin{array}{c}\text { simplistic } \\
\text { model }\end{array}$} \\
\hline & 1 & 2 & 0.125 & 1 & 2 & & \\
\hline $5 / 0.005$ & 86.92 & 82.59 & 104.32 & 86.93 & 82.58 & 64.08 & 64.0 \\
\hline $5 / 0.02$ & 149.94 & 143.42 & 165.17 & 147.78 & 143.43 & 143.77 & 143.20 \\
\hline $15.82 / 0.005$ & 136.90 & 130.38 & 152.13 & 139.09 & 130.40 & 120.30 & 119.92 \\
\hline
\end{tabular}

(a)
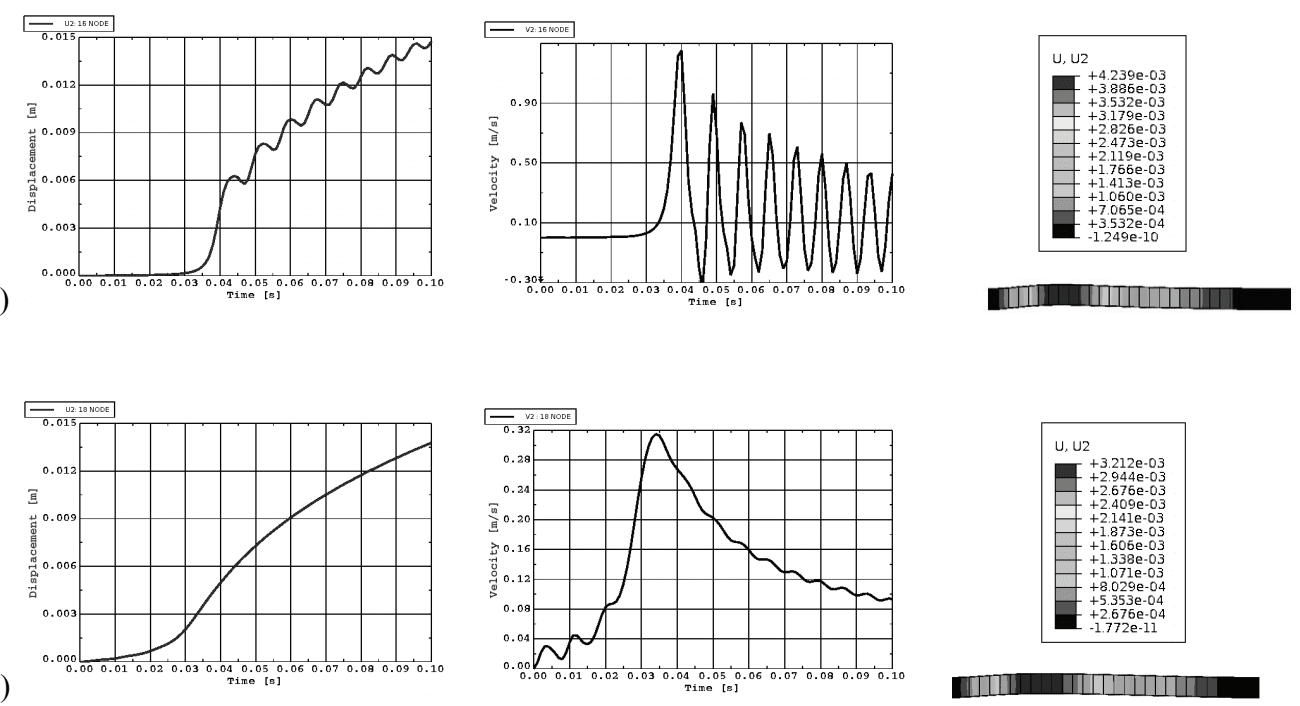

Fig. 8. Time histories of deflection and velocity of deflection for simplistic plate model with parameters: $G_{2}=5 \mathrm{MPa}, h_{2}=0.02 \mathrm{~m}$ and value $\xi_{2}$ equal to: (a) $\xi_{2}=0.125$, (b) $\xi_{2}=2$ 
The increase in values of number $\xi_{1}$ to $\xi_{1}=5,10$ in the axially-symmetrical component, expressed by term $\xi_{1}(\rho) \eta(\rho)$ in equation (3), decreases the values of critical dynamic loads $p_{\text {crdyn }}$ of plates with axiallysymmetrical mode $(m=0)$. However, the minimal values of critical dynamic load still correspond to circumferentially waved form of plate buckling. Figure 9 shows insignificant differences in the values of $p_{c r d y n}$ for plate modes with a small number of buckling waves $m=1,2$. More differences are observed for plate modes with greater number of buckling waves $m>4$. The marked tendency to increase in the value of $p_{c r d y n}$ for plates with preliminary deflection expressed by parameter $\xi_{1}=10$ is observed, too. The calculated minimal value of $p_{\text {crdyn }}$ corresponds to plate model with predeflection expressed by parameters: $m=0$ for $\xi_{1}=5$ and $m=5$ or $m=6$ for $\xi_{2}=1$ (see equation (3)). This value of $p_{c r d y n}$ is equal to $p_{c r d y n}=25.13 \mathrm{MPa}$. However, it is higher than the value calculated for plate without additional, axially-symmetrical component of preliminary deflection, when $\xi_{1}$ is equal to: $\xi_{1}=0$. The minimal value of $p_{c r d y n}$ is equal to: $p_{c r d y n}=$ $18.15 \mathrm{MPa}$.

The example calculations of FEM plate model with parameters $G_{2}=5 \mathrm{MPa}, h_{2}=0.005 \mathrm{~m}$ and $h^{\prime}=$ $0.001 \mathrm{~m}$, compressed on outer perimeter show some other interesting results. For all of the analysed examples of plate models with preliminary deformation described by parameters: $\xi_{1}=5, \xi_{2}=1$ and number of waves $m \neq 0$, excepting the cases of modes $m=2$ and $m=3$, there is observed the change of buckling form from the mode with number $m$ corresponding to the preliminary plate form to the form without the circumferential buckling waves. This occurs at the critical moment of plate loading. This observation is clearly shown in Fig. 10. In general, it could be observed that the differences in the values of critical parameters of plates compressed on outer edge with higher number of buckling mode are not noticeable. Existing decrease in values of critical dynamic loads for plates with smaller value of loading growth and the higher rate $\xi_{2}$ are for axisymmetric buckling mode of plates loaded on inner or outer edge. For plates loaded on inner edge, it is the minimal value of $p_{c r d y n}$.

Figure 11 shows a comparison of values $p_{c r d y n}$ for FDM and FEM basic plate models with parameters: $G_{2}=5 \mathrm{MPa}, h_{2}=0.005 \mathrm{~m}$ and calibrating numbers equal to: $\xi_{1}=0$ or $\xi_{1}=5$ and $\xi_{2}=1$ for the circumferentially waved plate cases, when the values of $p_{\text {crdyn }}$ are minimal. Presented results show that the disturbance of the geometry of preliminary plate shape by the additional axially-symmetrical component of equation (3) does not change the practically important final result. Still, the minimal value of critical dynamic load corresponds to plate mode with the same number of preliminary and expected buckling waves $m$. It is for $\xi_{1}=0$. Plates are loaded on outer perimeter with the rate of loading growth equal to $s \approx 931 \mathrm{MPa} / \mathrm{s}$ $(\mathrm{K} 7=201 / \mathrm{s})$ and $s=410.4 \mathrm{MPa} / \mathrm{s}$. The decrease in the value of rate $s$ additionally decreases the value of minimal critical dynamic loads $p_{c r d y n}$ calculated using the FEM. The values of critical dynamic loads $p_{c r d y n}$ of FDM plate models loaded with higher and lower $s$ parameter with wavy buckling forms $(m>4)$

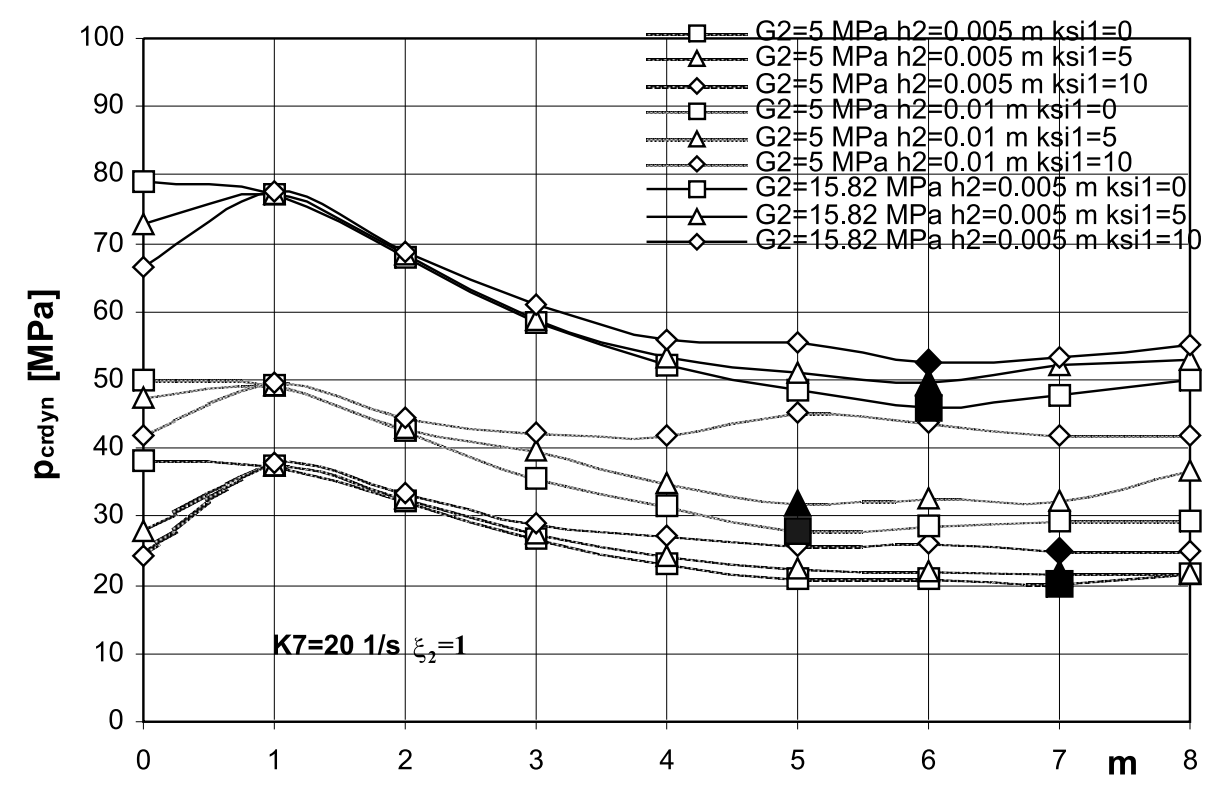

Fig. 9. The distribution of values of critical dynamic loads $p_{c r d y n}$ of plates with different material, geometric and $\xi_{1}$ values depending on the number of buckling waves $m$ 
do not change significantly. The minimal values of $p_{\text {crdyn }}$ for plates with preliminary geometry expressed by $\xi_{1}=0$ are similar.

Exemplary distributions of values of critical dynamic loads $p_{c r d y n}$ obtained using finite element method for plate with parameters $G_{2}=5 \mathrm{MPa}$ and $h_{2}=$ $0.005 \mathrm{~m}$ compressed on outer perimeter are presented in Fig. 12.

The results show the differences between the values of critical loads of plates loaded with various intensity and plates with different rate of imperfec- tion. The important minimal values of critical loads are for plates with parameters $\mathrm{K} 7=101 / \mathrm{s}$ and $\xi_{2}=2$. For these plates the values of $p_{c r d y n}$ are lower than the values of critical static loads $p_{c r}$. This confirms the importance of carrying out dynamic calculations.

The thickness of the sandwich plates' core is important in evaluating their dynamic behaviour [6]. The plates with thick core create some separate research group. In this work, these plate examples have the core thickness equal to $h_{2}=0.06 \mathrm{~m}$.
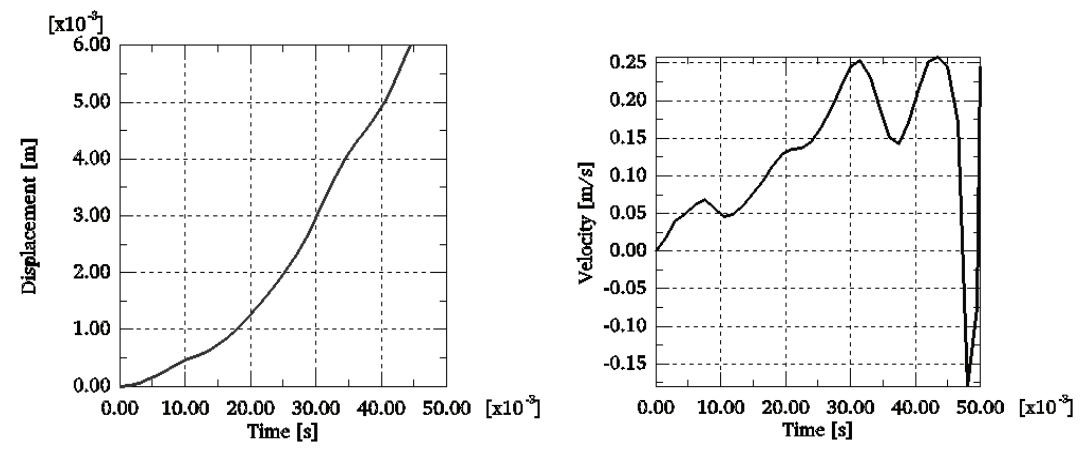

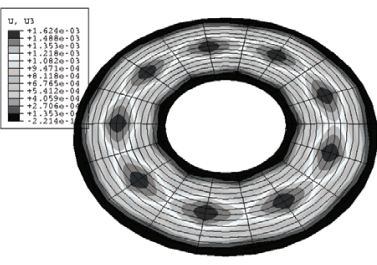

$t=0.0225 \mathrm{~s}$

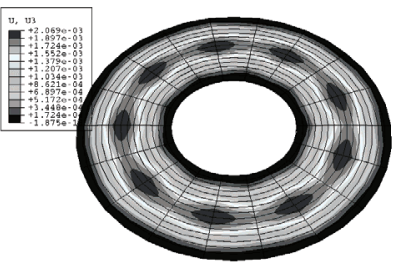

$t=0.0255 \mathrm{~s}$

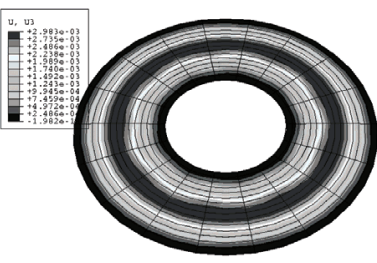

$t=0.03 \mathrm{~s}$

Fig. 10. Dynamic response of plate $\left(G_{2}=5 \mathrm{MPa}, h_{2}=0.005 \mathrm{~m}\right)$ compressed on outer perimeter with the parameters of preliminary deflection, equal to: $m=0$ for $\xi_{1}=5$ and $m=9$ for $\xi_{2}=1$

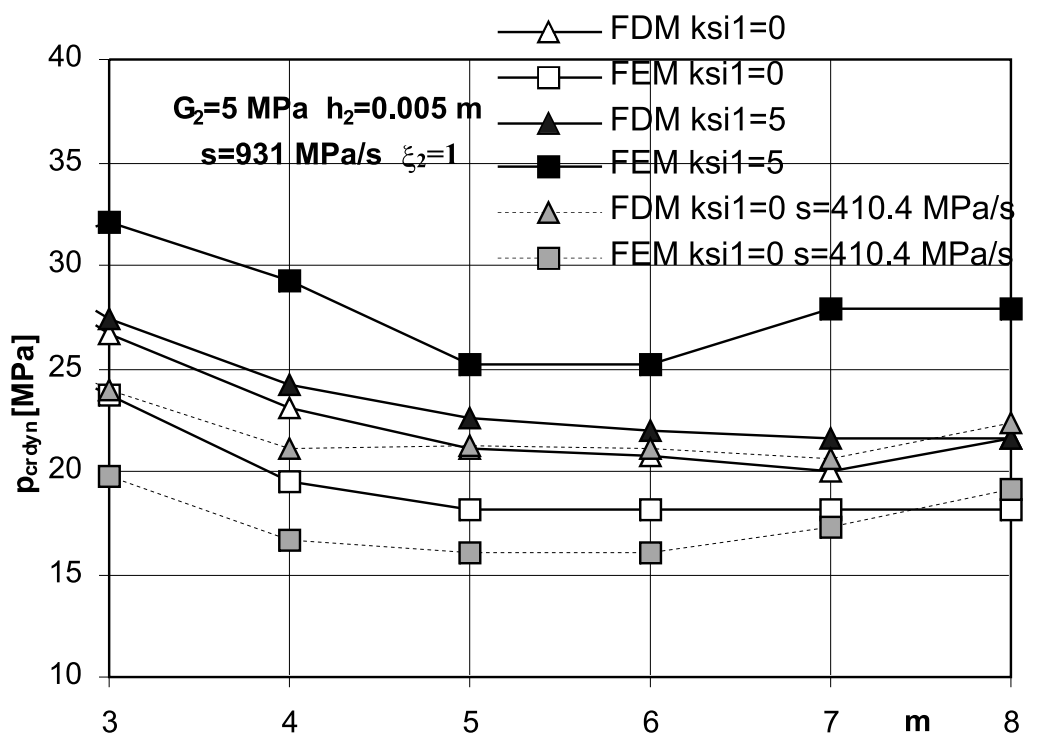

Fig. 11. The comparison of $p_{c r d y n}$ values for FDM and FEM plate models $\left(G_{2}=5 \mathrm{MPa}, h_{2}=0.005 \mathrm{~m}\right)$ with various imperfection parameter $\xi_{1}$ and loading intensity $s$ compressed on outer perimeter depending on the number of buckling waves $m$ 


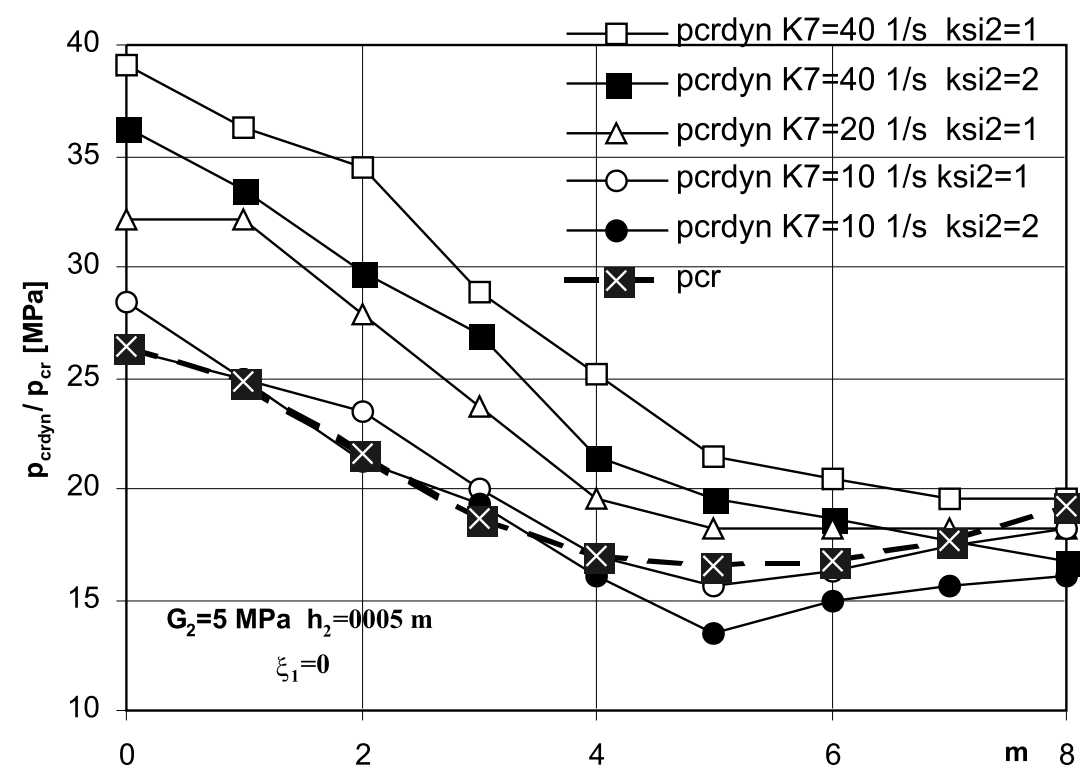

Fig. 12. Critical dynamic loads $p_{c r d y n}$ and static loads $p_{c r}$ distribution of FEM plate basic models with various $\mathrm{K} 7$ and $\xi_{2}$ parameters

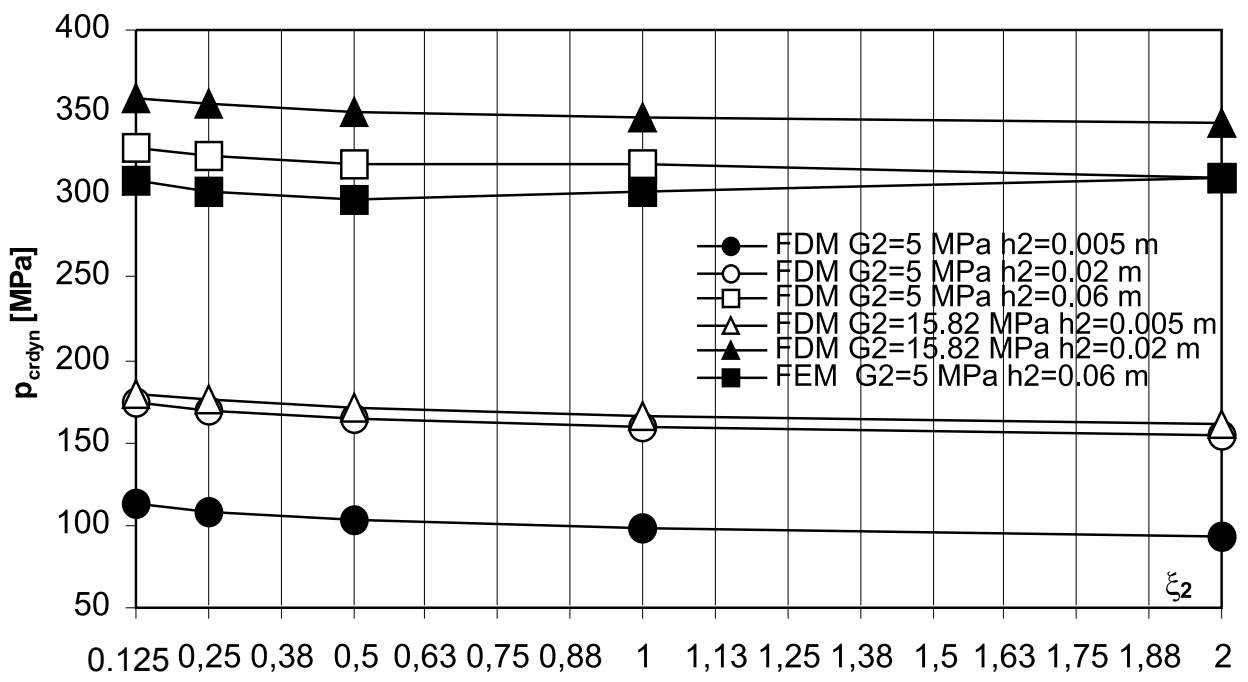

Fig. 13. Critical dynamic load $p_{c r d y n}$ distribution of FDM plate model and FEM simplistic one on inner edge depending on the rates of predeflection

The exemplary values of critical dynamic load $p_{\text {crdyn }}$ distribution depending on the rate of the plate preliminary defection $\xi_{2}\left(\xi_{1}=0\right)$ are presented in Fig. 13. The results are given for axisymmetrical buckling form $(m=0)$ of plates with various material and geometrical parameters radially compressed on inner edge. For each of the analysed examples of FDM plate model the decrease in values of critical dynamic loads $p_{\text {crdyn }}$ with the increase in numbers $\xi_{2}$ is observed. This observation is not confirmed by the results obtained for FEM simplistic plate model. Calculated values of $p_{c r d y n}$ for two extreme numbers $\xi_{2}=0.125$ and $\xi_{2}=2$ are comparable.

The decrease in loading is observed for number $\xi_{2}=0.5$, which, one can suspect, has the coinci- dental importance in this analysis. The time histories of deflections $\zeta_{1 \max }=f\left(t^{*}\right)$ for the FDM plate model confirm the observations (see Fig. 7) presented for plates with the middle core thickness. With the increase in the rate of predeflection supercritical vibrations disappear and the loss of plate stability occurs quicker.

Figure 14 shows the time histories of deflections, velocity of deflections and forms of critical deformations of FEM simplistic plate models with the rate of predeflection equal to: $\xi_{2}=0.125,0.5$. The loss of stability is in the global form. Qualitatively, supercritical vibrations distinguishes the plate case with the number $\xi_{2}=0.125$ from others. The small contraction 
(a)
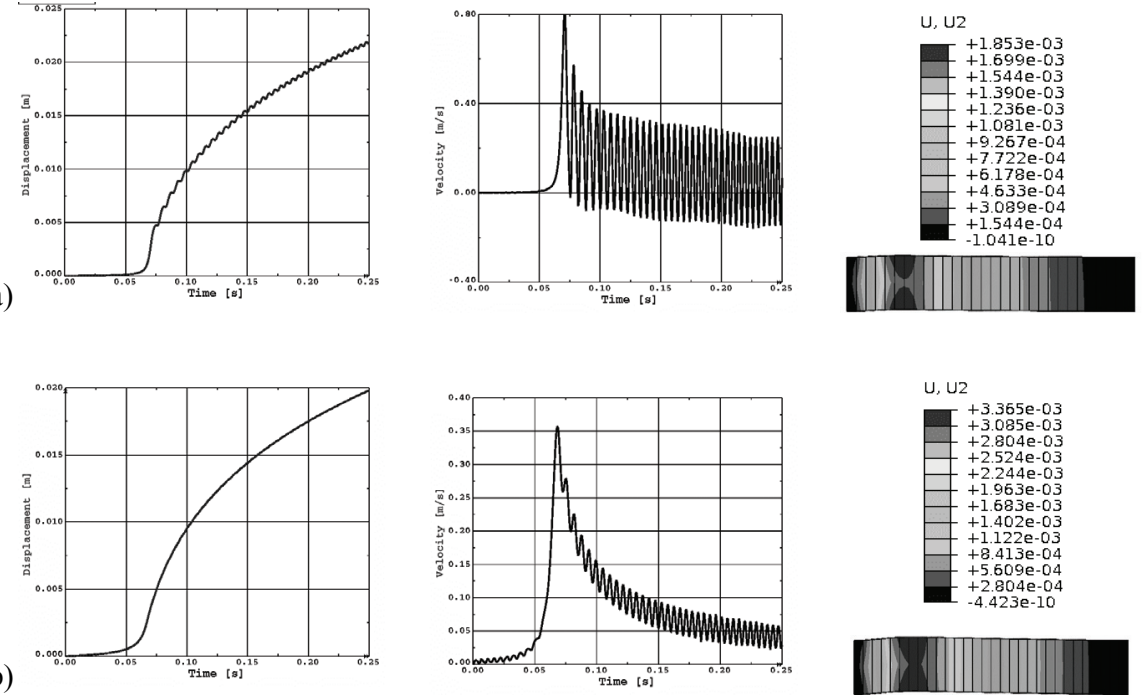

Fig. 14. Dynamic responses of FEM simplistic plate models $\left(G_{2}=5 \mathrm{MPa}, h_{2}=0.06 \mathrm{~m}, m=0\right)$ compressed on inner perimeter for the values of $\xi_{2}$ parameter, equal to: (a) $\xi_{2}=0.125$, (b) $\xi_{2}=0.5$

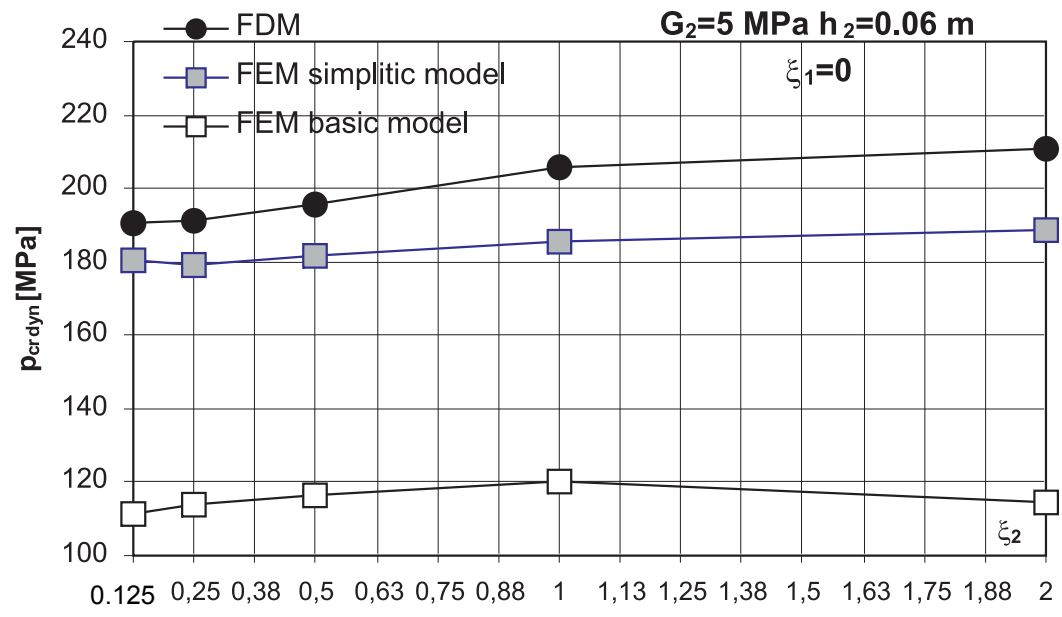

Fig. 15. Critical dynamic loads $p_{\text {crdyn }}$ distribution of FDM and FEM plate models compressed on outer edge depending on the rates of predeflection

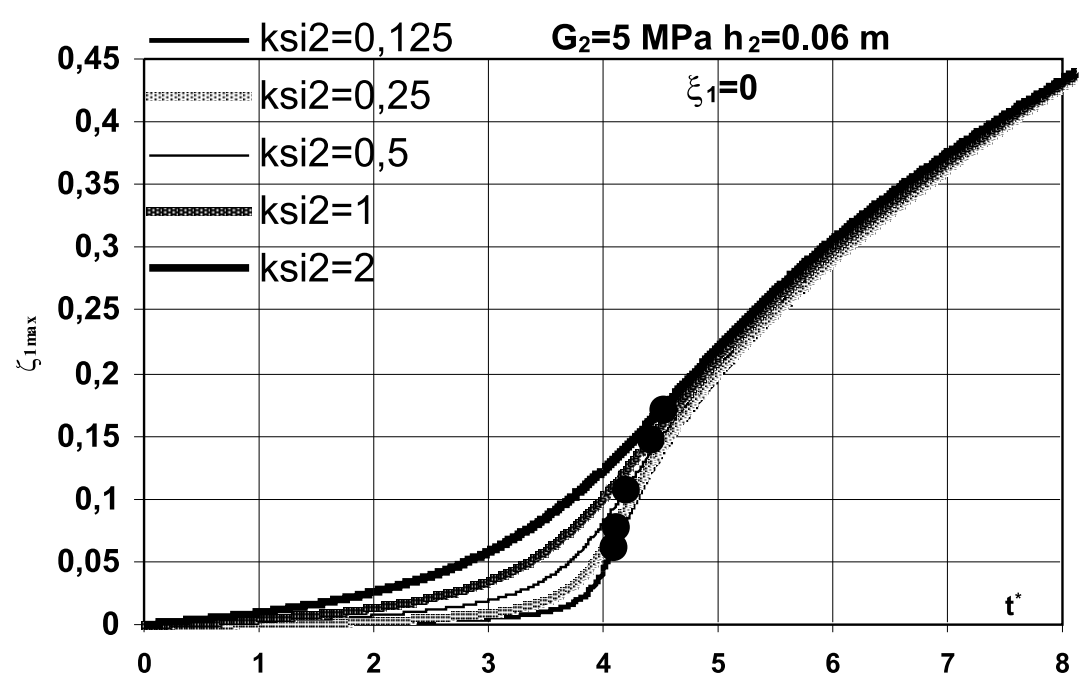

Fig. 16. Time histories of deflections of FDM plate model compressed on outer edge 
of the time $t_{c r}$ to the loss of plate stability and connected with it a decrease in the value of $p_{\text {crdyn }}$ is observed for the plate example with the number $\xi_{2}$ equal to $\xi_{2}=0.5$.

Figure 15 presents the results of axisymmetric $(m=0)$ plate compressed on outer edge. The rates of plate preliminary deflections are expressed by number $\xi_{2}$. The additional axisymmetric deformation does not exist $\xi_{1}=0$ (see equation (3)). The values of mode changes from axisymmetric $(m=0)$ to wavy ones in the critical region of plate work. The example results for plates with predeflection $\xi_{2}=0.125$ are shown in Fig. 17. The moment of the buckling form transformation corresponds with the critical regionpoint of the stability loss determined by accepted criterion. Existing disorder of the global form of the dynamic stability loss of plates with thick core is the typical observation for such plates. These qualitative
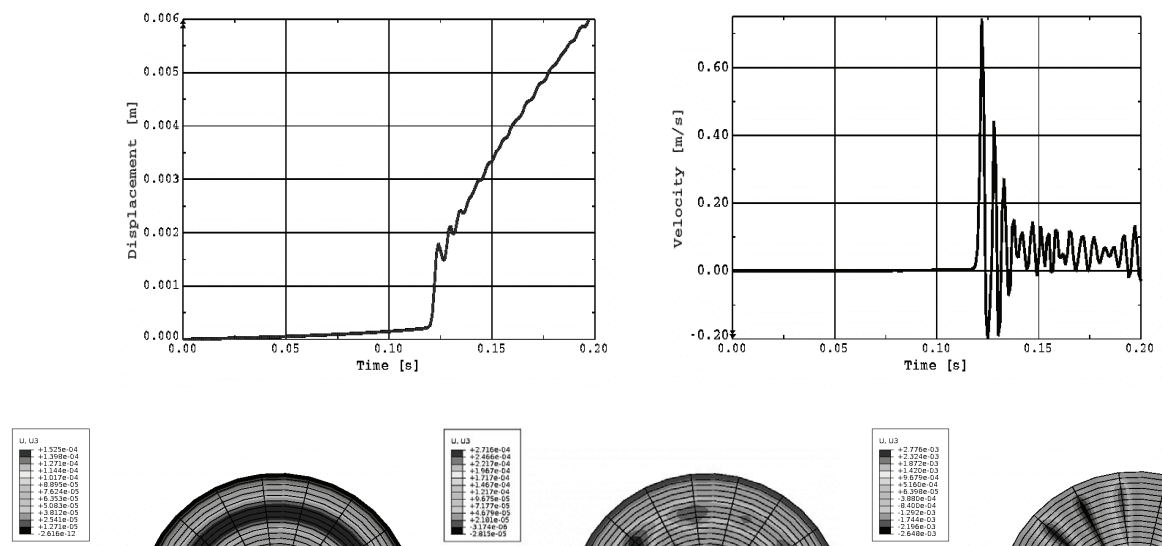

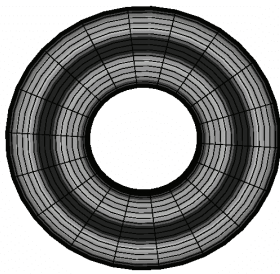

$t=0.10 \mathrm{~s}$

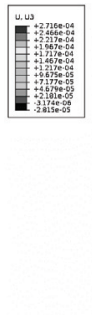

$t=0.12 \mathrm{~s}$

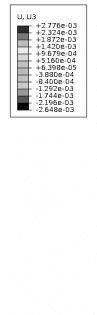

$t=0.14 \mathrm{~s}$

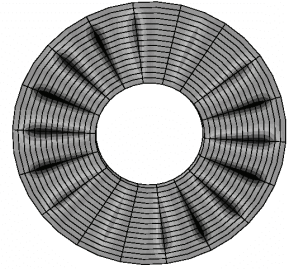

Fig. 17. Dynamic response of FEM plate model with parameters:

$G_{2}=5 \mathrm{MPa}, h_{2}=0.06 \mathrm{~m}, m=0, \xi_{1}=0, \xi_{2}=0.125$ compressed on outer perimeter

$p_{\text {crdyn }}$ calculated for FDM plate model and FEM simplistic one are approximated. In both cases, the decrease in values of $p_{\text {crdyn }}$ with increase in number $\xi_{2}$ is not observed. There is no relevant difference between the values of $p_{c r d y n}$ obtained for FEM basic plate models with numbers $\xi_{2}$ equal to: $\xi_{2}=0.125$ and $\xi_{2}=2$. Additionally, the values of $p_{\text {crdyn }}$ are much smaller than values calculates for FEM simplistic and FDM plate models.

Figure 16 shows the course of curves $\zeta_{1 \max }=f\left(t^{*}\right)$ of FDM plate models. The moment to the loss of plate dynamic stability, determined according to accepted stability criterion, prolongs and corresponding with it load $p_{c r d y n}$ increases for plate significantly preliminary deflected $\xi_{2}=2$. This observation differentiates such dynamic response of plate with thick core from other plates with middle core thickness (see Fig. 7) and also plates with thick core radially compressed on inner edge.

Additionally, the analyses of buckling deformation forms of FEM basic plate models show the changes of buckling mode could be a suitable meter of plate dynamic stability loss.

\section{CONCLUSIONS}

The numerical results presented show the degree of high sensitivity of plate sandwich structure to relatively little fluctuation in values of plate parameters, particularly including the preliminary deflections. In general, one can notice that the value of the critical dynamic load significantly decreases with the increase in the rate of plate predeflection. This decrease of critical value additionally rises when the speed of loading growth reduces.

Depending on the values of plate material and geometrical parameters the values of dynamic, critical loads could be lower than static ones. This shows that static loads cannot be the only one measure of the value order in quantity evaluation of plate critical 
loads. This fact confirms the necessity of conducting detailed computational plate investigations. The present analysis shows the importance of the general problem solution carried out for different forms of plate stability loss.

The observation that plates loaded on outer edge lose dynamic stability in circumferentially waved form for the minimal values of critical, dynamic loads, which are important in buckling problems, is the main conclusion of analysis. In general, there could be observed greater fluctuation of values of critical dynamic loads for plates with smaller number of circumferential waves $m<5$ and for plates with axisymmetric buckling form $m=0$. However, there are some problems with the formulation of the general principles of plate behaviour. The analyses presented show the difficulties with the evaluation of the plate dynamic, critical behaviour dependent on the many parameters and the important meaning of effective problem solution, which enables a relatively quick, numerical analysis of formulated task.

The analysis of dynamic behaviour of sandwich plates with thick core is not clear. This problem seems to require farther particular investigations. However, the obtained results of FEM basic plate model with the observed disorder of critical modes warn against imposing the global form of plate stability loss in analytical and numerical solution. Then, the values of critical loads could be admittedly inflated.

\section{REFERENCES}

[1] Chen Y.R., Chen L.W., Axisymmetric parametric resonance of polar orthotropic sandwich annular plates, Composite Structures, 2004, 65, 269-277.
[2] Chen Y.R., Chen L.W., Wang C.C., Axisymmetric dynamic instability of rotating polar orthotropic sandwich annular plates with a constrained damping layer, Composite Structures, 2006, 73(2), 290-302.

[3] CHEN Y.R., CHEN L.W., Vibration and stability of rotating polar orthotropic sandwich annular plates with a viscoelastic core layer, Composite Structures, 2007, 78, 45-57.

[4] Kluesener M.F., DraKe M.L., Mathematical Modelling. Damped Structure Design Using Finite Element Analysis, Shock and Vibration Bulletin, 1982, 52, 1-12.

[5] Majewski S., MaćKOWSKi R., Creep of foamed plastics used as the core of sandwich plate, Engineering and Building Industry, 1975, 3, 127-131, (in Polish).

[6] Pawlus D., Approach to evaluation of critical static loads of annular three-layered plates with various core thickness, Journal of Theoretical and Applied Mechanics, 2008, 46, 1, 85-107.

[7] PAwlus D., Dynamic stability of three-layered annular plates with viscoelastic core, Scientific Bulletin of the Technical University of Łódź, 2010, 1075, Łodź, (in Polish).

[8] Pawlus D., Dynamic stability of three-layered annular plates with wavy forms of buckling, Acta Mech., 2011, 216, 123-138.

[9] PAwlus D., Solution to the problem of axisymmetric and asymmetric dynamic instability of three-layered annular plates, Thin-Walled Structures, 2011, 49, 660-668.

[10] Romanów F., Strength of sandwich constructions, WSI in Zielona Gora, Poland, 1995, (in Polish).

[11] TROMBSKI M., WoJcIECH S., The cylindrically orthotropic annular plate subjected to time-dependent pressure acting in its plane, The Archive of Mechanical Engineering. 1981, XXVIII 2, 161-181, (in Polish).

[12] Volmir C., Stability of deformed system, Science, Moskwa, 1967, (in Russian).

[13] VolmiR C., Nonlinear dynamic of plates and shells, Science, Moskwa, 1972, (in Russian).

[14] WANG H.J., CHEN L.W., Vibration and damping analysis of a three-layered composite annular plate with a viscoelastic mid-layer, Composite Structures, 2002, 58, 563-570.

[15] WANG H.J., ChEN L.W., Axisymmetric dynamic stability of sandwich circular plates, Composite Structures, 2003, 59, 99-107.

[16] Wojciech S., Numerical solution of the problem of dynamic stability of annular plates, Journal of Theoretical and Applied Mechanics, 1979, 17, 2, 247-262, (in Polish). 